\title{
Inverse relationship between vitamin $D$ status and insulin resistance and the risk of impaired fasting glucose in Korean children and adolescents: the Korean National Health and Nutrition Examination Survey (KNHANES) 2009-2010
}

\author{
Seung Joon Chung ${ }^{1}$, Young Ah Lee ${ }^{1, *}$, Hyunsook Hong ${ }^{2}$, Min Jae Kang $^{1}$, \\ Hyun Jin Kwon ${ }^{3}$, Choong Ho Shin ${ }^{1}$ and Sei Won Yang \\ 'Division of Endocrinology and Metabolism, Department of Pediatrics, Seoul National University Children's \\ Hospital, Seoul National University College of Medicine, 101 Daehak-ro, Jongno-gu, Seoul 110-769, \\ Republic of Korea: ${ }^{2}$ Medical Research Collaborating Center, Seoul National University Hospital, Seoul, \\ Republic of Korea: ${ }^{3} \mathrm{Health}$ and Exercise Science, Institute of Sports Science, Seoul National University, \\ Seoul, Republic of Korea
}

Submitted 1 January 2013: Final revision received 31 May 2013: Accepted 25 July 2013: First published online 19 September 2013

\begin{abstract}
Objective: To investigate whether low vitamin D status was related to insulin resistance (IR) or impaired fasting glucose (IFG) in Korean adolescents, after adjusting for total body fat mass (FM).

Design: A cross-sectional study.

Setting: Korea National Health and Nutrition Examination Survey (KNAHNES) 2009-2010.

Subjects: In total, 1466 participants (769 males) aged 10-19 years were assessed for serum 25-hydroxyvitamin D (25(OH)D) levels, for FM by whole-body dualenergy X-ray absorptiometry and for IR by homeostasis model assessment (HOMA-IR) after an $8 \mathrm{~h}$ fast.

Results: Age-, sex-, season- and physical-activity-adjusted regression models showed that serum 25(OH)D levels were significantly related to markers of adiposity $(P=0.016$ for FM (g), $P=0.023$ for FM (\%) and $P=0.035$ for fat mass index). When the participants were stratified into three $25(\mathrm{OH}) \mathrm{D}$ categories $(<37 \cdot 5 \mathrm{nmol} / 1(n 553), 37 \cdot 5$ to $<50 \mathrm{nmol} / 1(n 543)$ and $\geq 50 \mathrm{nmol} / 1(n 370))$, significantly decreasing trends were observed for fasting insulin (all $P<0 \cdot 001$ ), HOMA-IR (all $P<0.001$ ) and the odds ratios for IFG (all $P$ for trend $<0.05$ ) from the lowest to the highest $25(\mathrm{OH}) \mathrm{D}$ category, after adjustments for age, sex, physical activity and all markers of adiposity. In the multivariate logistic regression analysis, the likelihood of participants in the lowest serum 25(OH)D category having IFG was $2 \cdot 96-3 \cdot 15$ compared with those in the highest $25(\mathrm{OH}) \mathrm{D}$ category (all $P<0 \cdot 05$ ).

Conclusions: There was a significant inverse relationship between vitamin D status and IR and the risk of IFG, independent of adiposity, in Korean adolescents.
\end{abstract}

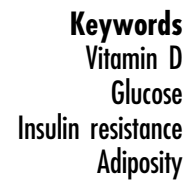

Low vitamin D (VitD) status is widespread ${ }^{(1,2)}$ and health concerns related to VitD insufficiency/deficiency are increasing. The VitD receptor is present on most cells of the body; VitD is required not only for bone health but may also play crucial roles in many diseases, including glucose metabolism, CVD, cancer and immune disorders ${ }^{(3)}$.

Low circulating 25-hydroxyvitamin D (25(OH)D) concentrations, an indicator of VitD status, have been linked to type 2 diabetes mellitus (T2DM), metabolic syndrome and other cardiometabolic risk factors in several adult studies ${ }^{(4-8)}$. Few epidemiological surveys have addressed paediatric populations ${ }^{(9,10)}$. There has been no population-based epidemiological report of VitD status, insulin resistance (IR) and $\mathrm{T} 2 \mathrm{DM}$ risk in an Asian paediatric population.

The mechanism by which VitD deficiency and T2DM risk are related is unclear. The theoretical evidence that VitD reduces $\mathrm{IR}^{(11-13)}$ and maintains insulin secretion ${ }^{(13-16)}$ supports the hypothesis that VitD reduces the risk of T2DM. However, VitD deficiency, impaired fasting glucose (IFG) and T2DM share the same risk factors, including African-American, Asian or Hispanic ethnicity, increased adiposity, older age and physical inactivity ${ }^{(16)}$. Therefore, adjustment must be made for these confounding factors 
in analyses of the association between VitD status and glucose homeostasis.

We investigated the relationships between VitD status and markers of IR and/or IFG using data based on the Korea National Health and Nutrition Examination Surveys (KNHANES) 2009-2010, after adjusting for age, sex, physical activity (PA) and adiposity. The adjusted markers of adiposity included BMI Z-score and total body fat measurements made with whole-body dual-energy X-ray absorptiometry (DXA).

\section{Materials and methods}

\section{Study population}

We used data from the third year (2009) of KNHANES IV and the first year (2010) of KNHANES V, with permission of the Korea Centers for Disease Control and Prevention. The KNHANES is a national survey based on a health interview survey, a nutrition survey and a health examination. KNHANES IV and V have a rolling sample design, with complex, stratified, multistage probability sampling. Among the 264186 primary sampling units (based on the 2005 National Census Registry), 200 and 192 sampling units were randomly selected, then twenty-three and twenty households were sampled from each unit, yielding 4600 and 3840 households in 2009 and 2010, respectively. In 2009 and 2010, 12722 and 10938 individuals were sampled, respectively: 10078 and 8473, respectively, participated in health interviews and health examinations; and 9397 and 8027, respectively, participated in the nutrition survey. Written informed parental consent and patient assent were obtained before testing. We analysed individuals who participated in KNHANES between 2009 and 2010 because these health examinations included measurements of body fat made with DXA since July 2009. Among the 2584 participants aged 10-19 years, 515 were excluded: those with missing fasting measures and/or those who had fasted for less than $8 \mathrm{~h}$ ( $n$ 262); those who had a previous history of diabetes ( $n$ 1); those whose fasting glucose level exceeded $7 \mathrm{mmol} / \mathrm{l}(n 2)$; and those with missing values for $25(\mathrm{OH}) \mathrm{D}$ ( $n$ 212) and/or fasting insulin or glucose ( $n$ 221). Among the 2069 participants, only 1472 were tested with DXA. There were no differences in sex, BMI $Z$-score, fasting blood glucose and insulin levels between adolescents who had DXA scans and those who did not. The following participants were also excluded: those who did not answer the PA questionnaire $(n$ 4) and/or those whose homeostatic model assessment of $\beta$-cell function (HOMA- $\beta$ ) and/or log transformation could not be calculated ( $n$ 2). In total, 1466 participants (769 males) were finally included.

Enrolment was conducted in all seasons: spring (March-May, $n$ 206), summer (June-August, $n$ 435), autumn (September-November, $n$ 438) and winter
(December-February, $n$ 387). Height, weight, BMI percentile and BMI $Z$-score were assigned on the basis of the 2007 Korean National Growth Charts ${ }^{(17)}$, and the participants were classified into three groups: lean ( $n$ 1158, $<85$ th BMI percentile); overweight ( $n$ 189, 85-95th BMI percentile); and obese ( $n$ 119, $\geq 95$ th BMI percentile). A questionnaire was used to assess the amount and intensity of PA performed. The participants were asked about their levels of PA during a normal week. The definition of 'regular PA' was based on the PA guideline provided by the US Department of Health and Human Services ${ }^{(18)}$ and the output indicators of the Korea Youth Risk Behavior Web-based Survey ${ }^{(19)}$. Participants who performed moderate or vigorous PA for at least $60 \mathrm{~min} / \mathrm{d}$ on $3 \mathrm{~d} /$ week, and/or walking activity for at least $60 \mathrm{~min} / \mathrm{d}$ on $5 \mathrm{~d} /$ week, and/or muscular strength activity on more than $3 \mathrm{~d}$ /week were assigned to the 'regular PA' group. Participants who performed moderate or vigorous PA for at least $180 \mathrm{~min} / \mathrm{d}$ on $3 \mathrm{~d}$ /week were also assigned to the 'regular PA' group.

\section{Laboratory measurements}

Blood samples were obtained by venepuncture, immediately refrigerated, transported to the central testing institute and analysed within $24 \mathrm{~h}$ of transportation. Serum 25(OH)D concentrations were measured with an immunoradiometric assay using a 1470 Wizard gammacounter (Perkin-Elmer, Turku, Finland) and a 25(OH)D ${ }^{125} \mathrm{I}$ radioimmunoassay kit (DiaSorin, Stillwater, MN, USA). At serum $25(\mathrm{OH}) \mathrm{D}$ levels of $21 \cdot 5,56 \cdot 8,82 \cdot 5$ and $122.5 \mathrm{nmol} / 1$, the inter-assay $\mathrm{CV}$ were $11.7 \%, 10.5 \%$, $8.6 \%$ and $12.5 \%$, respectively, and the intra-assay $\mathrm{CV}$ were $9 \cdot 4 \%, 8 \cdot 2 \%, 9 \cdot 1 \%$ and $11 \cdot 0 \%$, respectively. KNHANES participates in the VitD Standardization Program, so the measurement of $25(\mathrm{OH}) \mathrm{D}$ was standardized with the recently developed National Institute of Standards and Technology-Ghent University reference procedure ${ }^{(20)}$. Insulin levels were measured with a 1470 Wizard gammacounter and an immunoradiometric assay (Biosource, Fleurus, Belgium). Plasma glucose was measured enzymatically with a Hitachi Automatic Analyzer 7600 (Hitachi, Tokyo, Japan) and commercially available Pureauto S GLU kits (Sekisui, Tokyo, Japan). IR by homeostasis model assessment (HOMA-IR), HOMA- $\beta$ and the quantitative insulin-sensitivity check index (QUICKI) were calculated as previously described ${ }^{(21,22)}$.

\section{Total body fat measurements}

Whole-body DXA scans were performed with a QDR Discovery fan beam densitometer (Hologic, Inc., Bedford, MA, USA). The KNHANES data sets included DXA measurements of fat mass (FM) in grams. Body fatness is generally expressed as FM $(\mathrm{g})$ and FM as a percentage of body weight (FM (\%); calculated as $(\mathrm{FM} /$ total mass $) \times 100)$. A more appropriate approach is to normalize body fatness by height ${ }^{(23)}$. FM can be divided by height squared, to 
produce the fat mass index (FMI; calculated as (body FM excluding the head area)/height ${ }^{2}$ ). Until recently, no comprehensive source of body composition reference data for Korean children and adolescents was available. Therefore, we derived $Z$-scores for FM (g), FM (\%) and FMI from centile curves estimated using the LMS method ${ }^{(24)}$.

The DXA FM $Z$-scores were calculated as follows:

$$
Z=\left[(X / M)^{L}-1\right] /(L \times S),
$$

where $X$ is the DXA measurement for each individual, $L$ is the power transformation, $M$ is the median value and $S$ is the $\mathrm{CV}$ for each sex-age group centile curve.

\section{Statistical analysis}

All analyses were performed considering the KNHANES survey design and the weights used to ensure nationally representative estimates. The statistical software package IBM SPSS Statistics $19 \cdot 0$ was used to accommodate the complex sampling design with stratification, clustering and unequal weighting of the KNHANES sample. Statistical significance was defined as $P<0.05$ on two-sided tests.

All continuous variables are expressed as means with their standard errors and categorical variables as numbers and percentages of participants. When the distribution of a continuous variable was skewed, the variable was natural log-transformed for the analysis. The mean $25(\mathrm{OH}) \mathrm{D}$ concentrations according to subgroup were compared with an independent-samples $t$ test. Serum $25(\mathrm{OH}) \mathrm{D}$ concentrations were categorized into three groups: $<37 \cdot 5 \mathrm{nmol} / 1$ (the lowest category, $n 553$ ); $37 \cdot 5$ to $<50 \mathrm{nmol} / 1$ ( $n 543$ ); and $\geq 50 \mathrm{nmol} / 1$ (the reference group, $n$ 370). The Rao-Scott $\chi^{2}$ test was used to analyse the associations between the categorical variables and 25(OH) categories. The modifying effects of overweight status on the relationships between the three categories of $25(\mathrm{OH}) \mathrm{D}$ and the other dependent variables were evaluated (test for interaction). Tests for linear trends ( $P$ for linear trend) across the three categories of serum $25(\mathrm{OH}) \mathrm{D}$ were performed. We calculated the age- and sex-adjusted means for fasting glucose, fasting insulin, HOMA-IR, QUICKI and HOMA- $\beta$ according to the three categories of serum $25(\mathrm{OH}) \mathrm{D}$ and analysed these associations with a test for linear trends. Subgroup analyses were then performed to analyse the mean differences in the dependent variables between the reference category $(25(\mathrm{OH}) \mathrm{D} \geq 50 \mathrm{nmol} / \mathrm{l})$ and the other two categories.

Multivariate linear regression analyses were performed to assess the associations between the serum 25(OH)D categories and fasting glucose, fasting insulin, HOMA-IR, QUICKI and HOMA- $\beta$, after adjustment for potential confounders. Multivariable logistic regression analyses were used to assess the association between the $25(\mathrm{OH}) \mathrm{D}$ categories and the risk of IFG, after adjustment for potential confounders. Multivariate-adjusted odds ratios and $95 \%$ confidence intervals for the groups with
$25(\mathrm{OH}) \mathrm{D} \quad<37.5 \mathrm{nmol} / 1$ or 37.5 to $<50 \mathrm{nmol} / 1$ were computed by comparison with the reference category $(25(\mathrm{OH}) \mathrm{D} \geq 50 \mathrm{nmol} / \mathrm{l})$. We entered the potential confounders as follows: model 1 included age, sex, PA and BMI $Z$-score; model 2 included age, sex, PA, FM (g) Z-score; model 3 included age, sex, PA and FM (\%) $Z$-score; and model 4 included age, sex, PA and FMI $Z$-score.

\section{Results}

Table 1 presents the clinical characteristics of the study participants (mean age, $14 \cdot 3$ years). The $Z$-scores for height, weight and BMI for the total participants were 0.57 (SE 0.04), 0.31 (SE 0.04) and 0.08 (SE 0.04), respectively. The percentages of participants in the overweight and obese groups were $12 \cdot 9 \%$ and $8 \cdot 1 \%$, respectively. The mean level of serum $25(\mathrm{OH}) \mathrm{D}$ was $42 \cdot 0($ SE $0 \cdot 6) \mathrm{nmol} / \mathrm{l}$. The percentages of participants with serum 25(OH)D levels $<25 \mathrm{nmol} / 1$ ( $n$ 104), 25 to $<37.5 \mathrm{nmol} / 1$ ( $n$ 449), 37.5 to $<50 \mathrm{nmol} / 1$ ( $n$ 543), 50 to $<75 \mathrm{nmol} / 1$ ( $n$ 346) and $\geq 75 \mathrm{nmol} / 1$ ( $n$ 24) were $7 \cdot 1 \%, 30 \cdot 6 \%, 37 \cdot 1 \%, 23 \cdot 6 \%$ and $1 \cdot 6 \%$, respectively. The fasting glucose and insulin levels were 4.91 (SE 0.01) $\mathrm{mmol} / \mathrm{l}$ and 91.2 (se 1.5) pmol/l, respectively. Among the sixty-one ( $4.3 \%)$ participants with IFG $(5.6$ to $<7 \mathrm{mmol} / \mathrm{l})$, twenty-nine were overweight or obese.

There were significant differences in mean 25(OH)D levels between the subgroups based on age (10-14 $v$. 15-19 years), sex, season and regular PA, but not based on degree of obesity. In a univariate regression analysis, no significant relationships between the four markers of adiposity and serum 25(OH)D were observed. However, age-, sex-, season- and PA-adjusted regression models showed that serum $25(\mathrm{OH}) \mathrm{D}$ was significantly related to $Z$-scores for FM (g) $(P=0.016), \mathrm{FM}(\%)(P=0.023)$ and FMI $(P=0 \cdot 035)$, but not to $Z$-scores for BMI $(P=0 \cdot 265)$.

Table 2 shows the associations between the three categories stratified according to serum $25(\mathrm{OH}) \mathrm{D}$ and the participants' characteristics. Across the 25(OH)D categories, the participants in the highest $25(\mathrm{OH}) \mathrm{D}$ group were younger and more physically active than those in the lowest $25(\mathrm{OH}) \mathrm{D}$ group. The mean height $Z$-scores showed a significantly increasing trend $(P=0 \cdot 033)$ from the lowest to the highest $25(\mathrm{OH}) \mathrm{D}$ category. After adjustment for age and sex, significantly linear downward trends were observed for fasting glucose $(P=0 \cdot 039)$, fasting insulin $(P<0 \cdot 001)$, HOMA-IR $(P<0 \cdot 001)$ and HOMA- $\beta(P=0 \cdot 001)$ and an upward trend for QUICKI $(P<0 \cdot 001)$ with increasing $25(\mathrm{OH}) \mathrm{D}$ category (Table 2$)$. In the subgroup analysis, the age- and sex-adjusted means of fasting insulin, HOMA-IR and HOMA- $\beta$ were significantly lower in the reference category $(25(\mathrm{OH}) \mathrm{D}$ $\geq 50 \mathrm{nmol} / \mathrm{l})$ than in the other two categories of $25(\mathrm{OH}) \mathrm{D}$. The age- and sex-adjusted mean QUICKI for the reference category was significantly higher than those of the other two categories of $25(\mathrm{OH}) \mathrm{D}$ (Table 2). 
Table 1 Characteristics of study participants according to sex and 25(OH)D levels according to subgroups; adolescents aged 10-19 years, Korea National Health and Nutrition Examination Survey (KNHANES) 2009-2010

\begin{tabular}{|c|c|c|c|c|c|c|c|}
\hline & \multicolumn{2}{|c|}{ Total } & \multirow{2}{*}{$\frac{\text { Males }}{n}$} & \multirow{2}{*}{$\frac{\text { Females }}{n}$} & \multicolumn{2}{|c|}{ 25(OH)D (nmol/l) } & \multirow[b]{2}{*}{$P$ value } \\
\hline & $n$ & $\%$ & & & Mean & $\mathrm{SE}$ & \\
\hline Total participants & 1466 & 100 & 769 & 697 & $42 \cdot 6$ & 0.6 & \\
\hline \multicolumn{8}{|l|}{ Age (years) } \\
\hline $10-14$ & 804 & $54 \cdot 8$ & 431 & 373 & $44 \cdot 8$ & 0.7 & \multirow[t]{2}{*}{$<0.001$} \\
\hline $15-19$ & 662 & $45 \cdot 2$ & 338 & 324 & $39 \cdot 6$ & 0.8 & \\
\hline \multicolumn{8}{|l|}{ Sex } \\
\hline Males & 769 & $52 \cdot 5$ & 769 & 0 & $42 \cdot 8$ & 0.7 & \multirow[t]{2}{*}{0.021} \\
\hline Females & 697 & $47 \cdot 5$ & 0 & 697 & $41 \cdot 0$ & 0.7 & \\
\hline \multicolumn{8}{|l|}{ Season } \\
\hline Spring & 206 & $14 \cdot 1$ & 111 & 95 & $37 \cdot 4$ & $1 \cdot 0$ & \multirow[t]{4}{*}{$<0.01 t$} \\
\hline Summer & 435 & $29 \cdot 7$ & 230 & 205 & $47 \cdot 7$ & 0.9 & \\
\hline Autumn & 438 & $29 \cdot 8$ & 229 & 209 & $44 \cdot 3$ & 0.8 & \\
\hline Winter & 387 & $26 \cdot 4$ & 199 & 188 & $35 \cdot 6$ & 0.7 & \\
\hline \multicolumn{8}{|l|}{ Degree of obesity } \\
\hline Lean & 1158 & $79 \cdot 0$ & 598 & 560 & $41 \cdot 9$ & 0.6 & \multirow[t]{3}{*}{$0.25 \ddagger$} \\
\hline Overweight & 189 & $12 \cdot 9$ & 104 & 85 & $43 \cdot 1$ & $1 \cdot 1$ & \\
\hline Obese & 119 & $8 \cdot 1$ & 67 & 52 & $40 \cdot 8$ & $1 \cdot 7$ & \\
\hline \multicolumn{8}{|l|}{ WC $\geq 90$ th percentile } \\
\hline Yes & 126 & $8 \cdot 6$ & 61 & 65 & $41 \cdot 0$ & $1 \cdot 7$ & \multirow[t]{2}{*}{0.53} \\
\hline No & 1340 & $91 \cdot 4$ & 708 & 632 & $42 \cdot 1$ & 0.6 & \\
\hline \multicolumn{8}{|l|}{ Regular PA } \\
\hline Yes & 951 & 63.9 & 565 & 386 & $43 \cdot 6$ & 0.7 & \multirow[t]{2}{*}{$<0.001$} \\
\hline No & 515 & 36.1 & 204 & 311 & $39 \cdot 1$ & 0.7 & \\
\hline \multicolumn{8}{|l|}{ IFG } \\
\hline Yes & 61 & $4 \cdot 2$ & 31 & 30 & $40 \cdot 5$ & 1.9 & \multirow{6}{*}{$\begin{array}{l}0.43 \\
0.93\end{array}$} \\
\hline Lean & 32 & & 14 & 18 & $40 \cdot 7$ & $1 \cdot 4$ & \\
\hline Overweight or obese & 29 & & 17 & 12 & $40 \cdot 4$ & $3 \cdot 3$ & \\
\hline No & 1405 & $95 \cdot 8$ & 738 & 667 & $42 \cdot 0$ & 0.6 & \\
\hline HOMA-IR & 1466 & & 769 & 697 & $2 \cdot 92$ & 0.05 & \\
\hline QUICKI & 1466 & & 769 & 697 & 0.33 & 0.00 & \\
\hline
\end{tabular}

25(OH)D, 25-hydroxyvitamin D; WC, waist circumference; PA, physical activity; IFG, impaired fasting glucose; HOMA-IR, homeostatic model assessmentinsulin resistance index; QUICKI, quantitative insulin-sensitivity check index.

${ }^{*}$ Mean 25(OH)D concentrations according to subgroups were compared with an independent-samples $t$ test.

tEach mean 25(OH)D concentration according to subgroup was compared with the mean for the summer subgroup with an independent-samples $t$ test. $\ddagger$ A pairwise comparison was performed between all subgroups.

As further controls for adiposity, we used the BMI $Z$-scores (model 1) and body fat measurements made with DXA (model 2, FM (g); model 3, FM (\%); model 4, FMI). After adjustment for age, sex, PA and markers of adiposity, significantly decreasing trends for fasting insulin (all $P<0.001$ ), HOMA-IR (all $P<0.001$ ) and HOMA- $\beta(P=0.001$ for model 3 and $P=0.002$ for the others) and an increasing trend for QUICKI (all $P<0 \cdot 001$ ) were observed with increasing serum 25(OH)D (Table 3). After adjustment for HOMA-IR, there was no significant inverse relationship between HOMA- $\beta$ and the $25(\mathrm{OH}) \mathrm{D}$ categories. The modifying effects of overweight status on the relationship between the three categories of serum $25(\mathrm{OH}) \mathrm{D}$ and markers of IR were significant only for fasting insulin, HOMA-IR and QUICKI (test for interaction: $P=0.046, P=0.030$ and $P=0.034$, respectively). In the stratified analyses, $25(\mathrm{OH}) \mathrm{D}$ categories were significantly inversely related to fasting insulin and HOMA-IR, and positively related to QUICKI in both lean (all $P=0.001$ ) and overweight or obese participants (all $P<0 \cdot 001$; Table 3).

After adjustment for age, sex, PA and markers of adiposity (models 1-4), the multivariate-adjusted OR for IFG showed a significant decreasing trend with increasing
25(OH)D category (all $P$ for trend $<0 \cdot 05$, Fig. 1). The negative association between $25(\mathrm{OH}) \mathrm{D}$ categories and fasting glucose levels was marginally significant (Table 3). In the multivariate logistic regression analysis, the likelihood of participants in the lowest serum 25(OH)D category having IFG was $2 \cdot 96-3 \cdot 15$ compared with those in the highest $25(\mathrm{OH}) \mathrm{D}$ category (all $P<0.05$ for models 1-4, Fig. 1). No significant modifying effect of overweight status on the associations between $25(\mathrm{OH}) \mathrm{D}$ categories and fasting glucose (or the presence of IFG) or HOMA- $\beta$ was observed. In addition to body fat measurements (BMI $Z$-scores $(P=0 \cdot 001), \mathrm{FM}(\mathrm{g})(P<0 \cdot 001)$, FM (\%) $(P=0.006)$ and FMI $(P=0 \cdot 001))$, younger age $(P=0 \cdot 001)$ and less PA $(P<0 \cdot 05)$ were all significant predictors of IFG.

\section{Discussion}

The present nationally representative, population-based study showed that three-quarters of Korean adolescents aged 10-19 years were VitD deficient, and IR and the risk of IFG decreased significantly with increasing serum 
Table 2 Characteristics of study participants according to categories of serum 25(OH)D concentration; adolescents aged 10-19 years, Korea National Health and Nutrition Examination Survey (KNHANES) 2009-2010

\begin{tabular}{|c|c|c|c|c|c|c|c|}
\hline \multirow[b]{2}{*}{ Variable } & \multicolumn{2}{|c|}{$<37.5 \mathrm{nmol} / \mathrm{l}$} & \multicolumn{2}{|c|}{$37 \cdot 5$ to $<50 \mathrm{nmol} / \mathrm{l}$} & \multicolumn{2}{|c|}{$\geq 50 \mathrm{nmol}^{*}$} & \multirow[b]{2}{*}{$P$ value } \\
\hline & Mean & SE & Mean & SE & Mean & SE & \\
\hline Total participants $(n, \%)$ & 553 & $37 \cdot 7$ & 543 & $37 \cdot 1$ & 370 & $25 \cdot 2$ & \\
\hline Age (years) & $15 \cdot 6 \|$ & $0 \cdot 14$ & $14 \cdot 5$ & 0.16 & $14 \cdot 0$ & 0.22 & $<0.001$ \\
\hline $\operatorname{Sex}(n$ (males/female $))$ & \multicolumn{2}{|c|}{$265 / 288$} & \multicolumn{2}{|c|}{$285 / 258$} & \multicolumn{2}{|c|}{$219 / 151$} & $0.062^{\star *}$ \\
\hline Season ( $n$ (spring/summer/autumn/winter)) & \multicolumn{2}{|c|}{$112 / 92 / 111 / 238$} & \multicolumn{2}{|c|}{$74 / 162 / 194 / 113$} & \multicolumn{2}{|c|}{$20 / 181 / 133 / 36$} & $<0.001^{\star *}$ \\
\hline Height Z-score & 0.50 ศ & 0.060 & 0.56 & 0.071 & 0.69 & 0.062 & 0.033 \\
\hline Weight $Z$-score & 0.29 & 0.066 & 0.32 & 0.061 & 0.33 & $0 \cdot 056$ & 0.582 \\
\hline BMI Z-score & 0.07 & 0.069 & 0.09 & 0.055 & 0.06 & 0.063 & 0.917 \\
\hline FM (g) Z-score & 0.15 & 0.074 & $0 \cdot 15$ & 0.067 & 0.06 & 0.079 & 0.414 \\
\hline FM (\%) Z-score & 0.07 & 0.068 & $0 \cdot 10$ & 0.067 & -0.03 & 0.091 & 0.453 \\
\hline FMI Z-score & $0 \cdot 12$ & 0.074 & $0 \cdot 12$ & 0.068 & 0.00 & 0.086 & 0.320 \\
\hline Degree of obesity ( $n$ (lean/overweight/obese)) & \multicolumn{2}{|c|}{$435 / 68 / 50$} & \multicolumn{2}{|c|}{$427 / 69 / 47$} & \multicolumn{2}{|c|}{$296 / 52 / 22$} & $0 \cdot 440^{\star \star}$ \\
\hline WC $\geq 90$ th percentile $(n$ (no/yes)) & \multicolumn{2}{|c|}{$500 / 53$} & \multirow{2}{*}{\multicolumn{2}{|c|}{$\begin{array}{c}497 / 46 \\
188 / 355\end{array}$}} & \multicolumn{2}{|c|}{$343 / 27$} & $0.609^{\star \star}$ \\
\hline Regular PA ( $n$ (no/yes)) $\dagger$ & \multirow{2}{*}{\multicolumn{2}{|c|}{$\begin{array}{c}242 / 311 \\
523 / 30\end{array}$}} & & & \multirow{2}{*}{\multicolumn{2}{|c|}{$\begin{array}{l}85 / 285 \\
360 / 10\end{array}$}} & $<0.001^{* *}$ \\
\hline IFG $(n$ (no/yes) $) \ddagger$ & & & \multicolumn{2}{|c|}{$\begin{array}{l}188 / 355 \\
522 / 21\end{array}$} & & & $0.460^{\text {** }}$ \\
\hline Fasting blood glucose $(\mathrm{mmol} / \mathrm{l})$ & 4.94 & 0.02 & $4 \cdot 89$ & 0.02 & 4.89 & 0.02 & $0.039+t$ \\
\hline Fasting insulin $(\mathrm{pmol} / \mathrm{l}) \S$ & $96 \cdot 511$ & $2 \cdot 5$ & $92 \cdot 1 \|$ & 1.9 & $81 \cdot 0$ & $2 \cdot 0$ & $<0.001+t$ \\
\hline HOMA-IR $\$$ & $3 \cdot 116 \|$ & 0.086 & $2 \cdot 933 \|$ & 0.064 & $2 \cdot 575$ & 0.068 & $<0.001+t$ \\
\hline HOMA- $\beta \S$ & $198 \cdot 39 \|$ & $4 \cdot 732$ & $199 \cdot 2 \|$ & $4 \cdot 795$ & $173 \cdot 17$ & $4 \cdot 894$ & $0.001+t$ \\
\hline QUICKI\$ & $0 \cdot 329 \|$ & 0.001 & $0.330 \|$ & 0.001 & 0.336 & 0.001 & $<0.001+t$ \\
\hline
\end{tabular}

25(OH)D, 25-hydroxyvitamin D; FM, fat mass; WC, waist circumference; PA, physical activity; IFG, impaired fasting glucose; HOMA-IR, homeostatic model assessment-insulin resistance index; HOMA- $\beta$, homeostatic model assessment- $\beta$-cell function; QUICKI, quantitative insulin-sensitivity check index.

All continuous variables are given as means with their standard errors.

*Reference category.

tRegular PA ( $n$ (yes)) means the number of participants who performed moderate or vigorous physical activity for at least $60 \mathrm{~min} / \mathrm{d}$ on $3 \mathrm{~d} / \mathrm{week}$, and/or walking activity for at least $60 \mathrm{~min} / \mathrm{d}$ on $5 \mathrm{~d} / \mathrm{week}$, and/or muscular strength activity on more than $3 \mathrm{~d} / \mathrm{week}$, who were assigned to the 'regular physical activity' group. Those participants who performed moderate or vigorous physical activity for at least $180 \mathrm{~min} /$ day on $3 \mathrm{~d} / \mathrm{week}$ were also assigned to the 'regular physical activity' group. fIFG $(n(\mathrm{no}))$ means the number of participants with fasting glucose $<5.6 \mathrm{mmol} / \mathrm{l}$.

$\S$ The analysis was performed using natural log-transformed values.

IIThe value was significantly different from that of the reference category $(P<0.001)$.

The value was significantly different from that of the reference category $(P=0.027)$.

${ }^{\star *}$ The Rao-Scott $\chi^{2}$ test was used to analyse the associations between the categorical variables and the three categories of serum $25(\mathrm{OH}) \mathrm{D}$.

t+Tests for linear trend ( $P$ for linear trend) across the three categories of serum $25(\mathrm{OH}) \mathrm{D}$ levels were performed. We calculated the age- and sex-adjusted means for fasting glucose, fasting insulin, HOMA-IR, HOMA- $\beta$ and QUICKI according to the categories of serum 25(OH)D. Subgroup analyses were then performed to analyse the mean differences in the dependent variables between the reference category $(25(\mathrm{OH}) \mathrm{D} \geq 50 \mathrm{nmol} / \mathrm{l})$ and the other two categories.

Table 3 Multivariate linear regression analysis of the associations between serum 25(OH)D category (predictor variable) and fasting glucose, fasting insulin, HOMA-IR, HOMA- $\beta$ and QUICKI (outcomes); adolescents aged 10-19 years, Korea National Health and Nutrition Examination Survey (KNHANES) 2009-2010

\begin{tabular}{|c|c|c|c|c|c|c|c|c|c|c|c|c|}
\hline \multirow[b]{2}{*}{ Dependent variable } & \multicolumn{3}{|c|}{ Model 1} & \multicolumn{3}{|c|}{ Model 2} & \multicolumn{3}{|c|}{ Model 3} & \multicolumn{3}{|c|}{ Model 4} \\
\hline & $\beta$ & $\mathrm{SE}$ & $P$ value & $\beta$ & SE & $P$ value & $\beta$ & $\mathrm{SE}$ & $P$ value & $\beta$ & $\mathrm{SE}$ & $P$ value \\
\hline \multicolumn{13}{|l|}{ Total participants $(n 1$} \\
\hline Fasting glucose & -0.473 & 0.245 & 0.054 & -0.462 & 0.245 & 0.061 & -0.484 & $0 \cdot 246$ & 0.050 & -0.463 & 0.245 & 0.060 \\
\hline Fasting insulin* & -0.064 & 0.014 & $<0.001$ & -0.063 & 0.014 & $<0.001$ & -0.067 & 0.014 & $<0.001$ & -0.063 & 0.013 & $<0.001$ \\
\hline HOMA-IR & -0.069 & 0.015 & $<0.001$ & -0.068 & 0.014 & $<0.001$ & -0.072 & 0.014 & $<0.001$ & -0.068 & 0.014 & $<0.001$ \\
\hline QUICKI* & 0.010 & 0.002 & $<0.001$ & 0.010 & 0.002 & $<0.001$ & 0.010 & 0.002 & $<0.001$ & 0.010 & 0.002 & $<0.001$ \\
\hline HOMA- $\beta^{*}$ & -0.048 & 0.015 & 0.002 & -0.047 & 0.015 & 0.002 & -0.050 & 0.015 & 0.001 & -0.048 & 0.015 & 0.002 \\
\hline HOMA- $\beta+$ & -0.006 & 0.012 & 0.629 & -0.005 & 0.012 & 0.666 & -0.005 & 0.012 & 0.674 & -0.005 & 0.012 & 0.673 \\
\hline \multicolumn{13}{|l|}{ Lean participants ( } \\
\hline Fasting insulin* & -0.045 & 0.013 & $<0.001$ & -0.044 & 0.013 & 0.001 & -0.047 & 0.013 & 0.001 & -0.045 & 0.013 & 0.001 \\
\hline HOMA-IR* & -0.048 & 0.014 & 0.001 & -0.047 & 0.014 & 0.001 & -0.049 & 0.014 & 0.001 & -0.048 & 0.014 & 0.001 \\
\hline QUICKI* & 0.007 & 0.002 & 0.001 & 0.007 & 0.002 & 0.001 & 0.007 & 0.002 & 0.001 & 0.007 & 0.002 & 0.001 \\
\hline \multicolumn{13}{|c|}{ Overweight or obese participants ( $n 308$ ) } \\
\hline Fasting insulin* & -0.149 & 0.038 & $<0.001$ & $-0 \cdot 155$ & 0.036 & $<0.001$ & $-0 \cdot 159$ & 0.036 & $<0.001$ & -0.154 & 0.037 & $<0.001$ \\
\hline HOMA-IR* & $-0 \cdot 163$ & 0.040 & $<0.001$ & $-0 \cdot 170$ & 0.038 & $<0.001$ & $-0 \cdot 174$ & 0.037 & $<0.001$ & $-0 \cdot 168$ & 0.038 & $<0.001$ \\
\hline QUICKI* & 0.022 & 0.005 & $<0.001$ & 0.023 & 0.005 & $<0.001$ & 0.024 & 0.005 & $<0.001$ & 0.023 & 0.005 & $<0.001$ \\
\hline
\end{tabular}

25(OH)D, 25-hydroxyvitamin D; HOMA-IR, homeostatic model assessment-insulin resistance index; HOMA- $\beta$, homeostatic model assessment- $\beta$-cell function; QUICKI, quantitative insulin-sensitivity check index.

Multivariate linear regression analyses were performed to assess the association between increasing $25(\mathrm{OH}) \mathrm{D}$ category $(<37 \cdot 5 \mathrm{nmol} / \mathrm{l}, 37 \cdot 5$ to $<50 \mathrm{nmol} / \mathrm{l}$, $\geq 50 \mathrm{nmol} / \mathrm{I}$ ) and the change in fasting glucose, fasting insulin, HOMA-IR, HOMA- $\beta$ and QUICKI, after adjustment for potential confounders. If there was an interaction between the three categories of serum $25(\mathrm{OH}) \mathrm{D}$ and BMI Z-scores (<85th percentile or $\geq 85$ th percentile), we split the group into two (lean $v$. overweight/obese). The modifying effect of overweight status on the relationship between the three categories of serum 25(OH)D and markers of insulin resistance was significant (test for interaction: $P=0.046$ for fasting insulin, $P=0.030$ for HOMA-IR and $P=0.034$ for QUICKI). No significant modifying effect of overweight status on the association between the $25(\mathrm{OH}) \mathrm{D}$ categories and fasting glucose or HOMA- $\beta$ was observed. We entered potential confounders as follows: model 1 included age, sex, physical activity (PA) and BMI Z-score; model 2 included age, sex, PA and fat mass (g) Z-score; model 3 included age, sex, PA and fat mass (\%) Z-score; and model 4 included age, sex, PA and fat mass index Z-score.

*The analysis was performed using natural log-transformed values for the dependent variables.

tThe analysis was performed after additional adjustment of natural log-transformed HOMA-IR. 


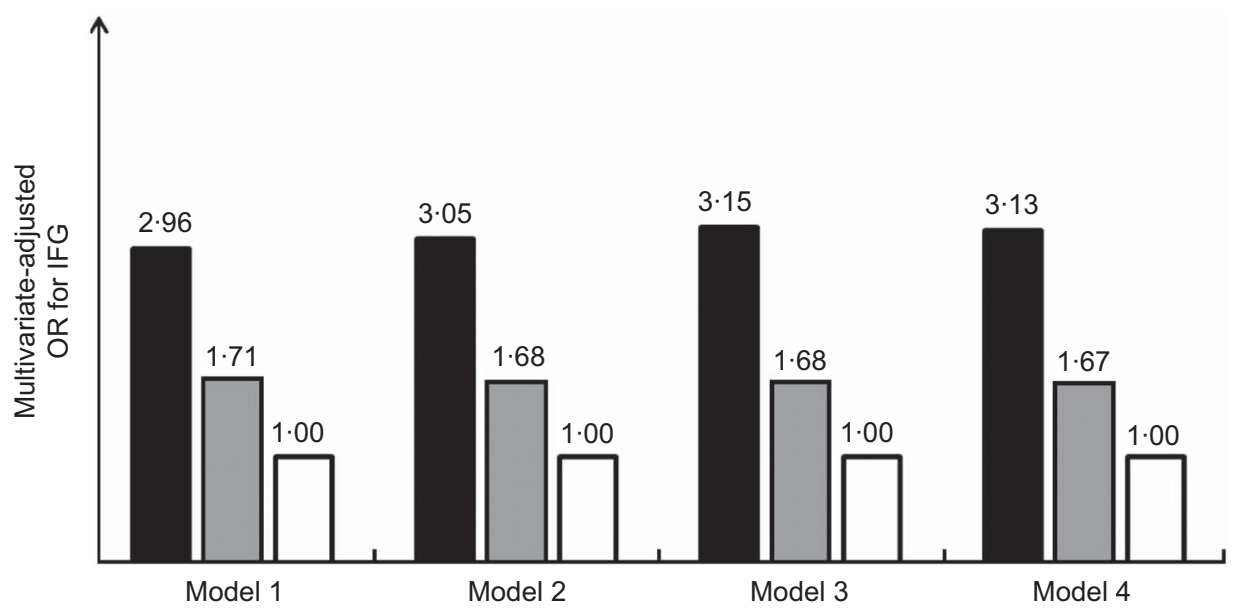

Figure 1 Multivariate-adjusted odds ratios for impaired fasting glucose (IFG) according to serum 25-hydroxyvitamin D level $(\square,<37.5 \mathrm{nmol} / \mathrm{l}, n 553 ; \square, 37 \cdot 5$ to $<50 \mathrm{nmol} / \mathrm{l}, n 543 ; \square, \geq 50 \mathrm{nmol} / \mathrm{l}, n 370)$ among adolescents aged $10-19$ years, Korea National Health and Nutrition Examination Survey (KNHANES) 2009-2010. Model 1 adjusted for age, sex, physical activity (PA) and BMI $Z$-score; model 2 for age, sex, PA and fat mass (g) Z-score; model 3 for age, sex, PA and fat mass (\%) Z-score; and model 4 for age, sex, PA and fat mass index Z-score

25(OH)D, independently of age, sex, PA and adiposity. To our knowledge, this is the first nationally representative study suggesting a significant relationship between VitD status and markers of glucose homeostasis, even after adjustment for total body fat measurements (made with DXA), in an Asian paediatric population.

The potential effects of VitD on glucose homeostasis are considered to be mediated by autocrine and paracrine functions involving the transcriptional regulation of genes in pancreatic $\beta$-cells, skeletal myocytes and immune cells, improving insulin secretion and sensitivity and reducing inflammation $^{(13,16)}$. Despite theoretical links between VitD and insulin sensitivity and/or secretion, controversy remains regarding the relationship between $25(\mathrm{OH}) \mathrm{D}$ levels and surrogate indices of insulin sensitivity ${ }^{(4-7,25,26)}$ or $\beta$-cell function ${ }^{(7,25,26)}$ and the risk of glucose intolerance or $\mathrm{T} 2 \mathrm{DM}^{(6,9,27)}$. These discrepancies in the relationships between VitD status and outcomes related to glucose homeostasis in paediatric or adult populations may be attributable to differences in study sample size, participant characteristics (race, ethnicity and degree of obesity), method of measuring insulin sensitivity and/or $\beta$-cell function, and the adjusted markers of adiposity used.

Adipose tissue is not only the natural reservoir of liposoluble $25(\mathrm{OH}) \mathrm{D}$, but also a well-known determinant of IR. Traditional measurements, such as BMI, do not measure fat in accurate quantitative terms ${ }^{(23)}$. However, a technique that can divide body weight into FM and fat-free mass (such as DXA) is useful in adjusting for total body adiposity. We first investigated the association between total body fat and serum 25(OH)D and then evaluated whether serum $25(\mathrm{OH}) \mathrm{D}$ was related to surrogate indices of insulin sensitivity (HOMA-IR, QUICKI) or insulin secretion (HOMA- $\beta$ ), and the risk of IFG, after adjusting for total body adiposity using DXA.
Racial and ethnic differences in the degree of adiposity and the distribution of body fat are well known ${ }^{(28,29)}$. Asian-American and African-American children have lower BMI and/or total body fat than Caucasian children. Asian-American children have higher visceral adipose tissue $^{(29)}$ and African-American children have lower visceral adipose tissue and more subcutaneous adipose tissue than Caucasian children ${ }^{(28)}$. Lower levels of $25(\mathrm{OH}) \mathrm{D}$ are associated with higher adiposity, measured with DXA, in Caucasian and African-American youth examined concurrently, and VitD deficiency is associated with higher visceral adipose tissue in Caucasians and greater subcutaneous adipose tissue in African-Americans ${ }^{(28)}$. We demonstrated a significant association between serum 25(OH)D levels and total body fat, measured with DXA, after adjustment for age, sex, season and PA in a Korean paediatric population, although we could not examine the relationship between $25(\mathrm{OH}) \mathrm{D}$ status and the distribution of abdominal adipose tissue.

Theoretically, a VitD response element in the human insulin receptor gene promoter might affect insulin receptor expression ${ }^{(11)}$, and 1,25-dihydroxycholecalciferol (1,25-dihydroxyvitamin $\mathrm{D}_{3}$ ) directly stimulates its expression and insulin responsiveness for glucose transport ${ }^{(12)}$. VitD also indirectly improves insulin responsiveness by maintaining normal extracellular calcium levels and effective calcium influx through cell membranes ${ }^{(13)}$. Many cross-sectional studies have supported an association between 25(OH)D levels and insulin sensitivity in adults $^{(4-6,8)}$. Recently, Gagnon et al. ${ }^{(4)}$ prospectively demonstrated that lower $25(\mathrm{OH}) \mathrm{D}$ levels are associated with increased HOMA-IR and risk of metabolic syndrome in Australian adults. In cross-sectional studies of Chinese ${ }^{(5)}$ and Korean adults ${ }^{(6)}$, VitD status was inversely related to HOMA-IR. LOw 25(OH)D levels are also significantly 
related to an increased risk of metabolic syndrome in Chinese adults ${ }^{(5)}$ and T2DM in Korean adults ${ }^{(6)}$. Consistent with studies of adult populations ${ }^{(4-6,8)}$, cross-sectional nationwide studies of paediatric populations have consistently reported an inverse association between $25(\mathrm{OH}) \mathrm{D}$ levels and fasting hyperglycaemia and/or increased HOMA-IR, after adjustment for BMI ${ }^{(9,10)}$. Our study supports a significant relationship between $25(\mathrm{OH}) \mathrm{D}$ levels and HOMA-IR or QUICKI, independent of total body fat. The inverse relationship between $25(\mathrm{OH}) \mathrm{D}$ and IR was significant in all participants, not only those who were overweight or obese.

VitD receptors ${ }^{(14)}$ and $1 \alpha$-hydroxylase ${ }^{(15)}$ are present in pancreatic $\beta$-cells and calcium plays an essential role in $\beta$-cell insulin secretion ${ }^{(13,16)}$. Therefore, VitD may directly or indirectly affect insulin secretion. Contrary to expectation, HOMA- $\beta$ showed an increasing trend with decreased serum $25(\mathrm{OH}) \mathrm{D}$, which was not significant after adjustment for HOMA-IR. Therefore, the failure of $\beta$-cell mass compensation for decreased insulin sensitivity related to VitD deficiency and/or other risk factors may be associated with the development of fasting hyperglycaemia or IFG.

The risk of diabetes associated with IR and $\beta$-cell compensation varies with race/ethnicity. Compared with Caucasians, total adiposity is lower, but the risk of incident diabetes is 18\% higher in Asian-Americans ${ }^{(29)}$, which may be linked to their greater abdominal obesity, smaller muscle mass and lower $\beta$-cell insulin secretion in the context of $\operatorname{IR}^{(29,30)}$. Unexpectedly, about half of the participants with IFG in our study were lean. The lean participants with IFG may have had a 'metabolically obese, but normal-weight' phenotype (lean individuals with a disproportionate amount of fat stored within the abdomen) and/or a family history of T2DM. Unfortunately, the KNHANES data did not include body fat topography measurements and/or complete answers to the family history questionnaire for the paediatric population. Although there is insufficient evidence to recommend consistent thresholds of serum $25(\mathrm{OH}) \mathrm{D}$ in relation to noncalcaemic metabolic benefits, numerous epidemiological studies have suggested that $25(\mathrm{OH}) \mathrm{D}>75 \mathrm{nmol} / \mathrm{l}$ may confer health benefits by reducing the risk of T2DM, CVD, etc. ${ }^{(31)}$. Fewer than $2 \%$ of our participants were VitD sufficient $(>75 \mathrm{nmol} / \mathrm{l})$, irrespective of their overweight status. VitD insufficiency and/or deficiency may be related to hepatic IR, and the $\beta$-cell mass cannot fully compensate or overcome IR in Korean adolescents.

The study was limited by a lack of information about the participants' pubertal status and family history of T2DM, levels of parathyroid hormone and/or several adipocytokines, and visceral fat measurements. We used HOMA-IR and QUICKI, reflecting only hepatic insulin sensitivity rather than in vivo whole-body insulin sensitivity, which encompasses both hepatic and peripheral tissues, measured using hyperinsulinaemic-euglycaemic and hyperglycaemic clamps. Rajakumar et al. ${ }^{(26)}$ recently reported that $25(\mathrm{OH}) \mathrm{D}$ levels are not independently related to in vivo insulin sensitivity or $\beta$-cell function, after adjustment for total body fat or visceral adipose tissue, in healthy black and white youth aged 8-18 years without dysglycaemia. Our study is the first to show that a low VitD status is significantly related to hepatic IR and IFG, independently of total body fat, in Korean adolescents. Further studies are required to investigate whether the association between $25(\mathrm{OH}) \mathrm{D}$ status and the parameters of glucose homeostasis is significant in Korean adolescents after adjustment for visceral adiposity, which determines metabolic obesity in Asian populations.

Unexpectedly, the mean height $Z$-score showed an increasing trend with increasing $25(\mathrm{OH}) \mathrm{D}$. Serum $25(\mathrm{OH}) \mathrm{D}$ levels were positively related to final adult height in ninety young women ${ }^{(32)}$. Adolescent girls with VitD deficiency showed a significant reduction in height ${ }^{(33)}$. Because of its essential role in skeletal development and bone health, VitD may have a beneficial effect on linear growth and final height.

\section{Conclusion}

Low VitD status was significantly associated with hepatic IR and IFG, independently of total body fat, in Korean adolescents. It remains to be determined whether VitD optimization improves insulin sensitivity and reverses IFG in VitD-deficient adolescents, irrespective of changes in their total or visceral adiposity.

\section{Acknowledgements}

Sources of funding: This research received no specific grant from any funding agency in the public, commercial or not-for-profit sectors. There was no funder's contribution to the study design, conduct of the study, analysis of samples or data, interpretation of findings or the preparation of this manuscript. Conflicts of interest: There are no conflicts of interest for any author. Ethics: The study complied with the recommendations of the Declaration of Helsinki. The institutional review board of the Korea Centers for Disease Control and Prevention approved the study protocol. Authors' contributions: S.J.C., Y.A.L., M.J.K., S.W.Y. and C.H.S. were responsible for the conception and design of the study. S.J.C., Y.A.L., H.S.H. and H.J.K. contributed to preparation of the data set. S.J.C., Y.A.L. and H.S.H. performed the statistical analysis. S.J.C. and Y.A.L. drafted the manuscript. All authors played a role in interpretation of the results and approved the final manuscript.

\section{References}

1. Kumar J, Muntner P, Kaskel FJ et al. (2009) Prevalence and associations of 25-hydroxyvitamin D deficiency in US children: NHANES 2001-2004. Pediatrics 124, e362-e370. 
2. Choi HS, Oh HJ, Choi H et al. (2011) Vitamin D insufficiency in Korea - a greater threat to younger generation: the Korea National Health and Nutrition Examination Survey (KNHANES) 2008. J Clin Endocrinol Metab 96, 643-651.

3. Bouillon R, Carmeliet G, Verlinden L et al. (2008) Vitamin D and human health: lessons from vitamin $\mathrm{D}$ receptor null mice. Endocr Rev 29, 726-776.

4. Gagnon C, Lu ZX, Magliano DJ et al. (2012) Low serum 25-hydroxyvitamin $\mathrm{D}$ is associated with increased risk of the development of the metabolic syndrome at five years: results from a national, population-based prospective study (The Australian Diabetes, Obesity and Lifestyle Study: AusDiab). J Clin Endocrinol Metab 97, 1953-1961.

5. Lu L, Yu Z, Pan A et al. (2009) Plasma 25-hydroxyvitamin D concentration and metabolic syndrome among middleaged and elderly Chinese individuals. Diabetes Care 32, $1278-1283$.

6. Choi HS, Kim KA, Lim CY et al. (2011) Low serum vitamin D is associated with high risk of diabetes in Korean adults. J Nutr 141, 1524-1528.

7. Kayaniyil S, Retnakaran R, Harris SB et al. (2011) Prospective associations of vitamin $\mathrm{D}$ with $\beta$-cell function and glycemia: the PROspective Metabolism and ISlet cell Evaluation (PROMISE) cohort study. Diabetes 60, 2947-2953.

8. Gagnon C, Lu ZX, Magliano DJ et al. (2011) Serum 25-hydroxyvitamin D, calcium intake, and risk of type 2 diabetes after 5 years: results from a national, populationbased prospective study (the Australian Diabetes, Obesity and Lifestyle Study). Diabetes Care 34, 1133-1138.

9. Reis JP, von Muhlen D, Miller ER et al. (2009) Vitamin D status and cardiometabolic risk factors in the United States adolescent population. Pediatrics 124, e371-e379.

10. Ganji V, Zhang X, Shaikh $\mathrm{N}$ et al. (2011) Serum 25-hydroxyvitamin D concentrations are associated with prevalence of metabolic syndrome and various cardiometabolic risk factors in US children and adolescents based on assay-adjusted serum 25-hydroxyvitamin D data from NHANES 2001-2006. Am J Clin Nutr 94, 225-233.

11. Maestro B, Davila N, Carranza MC et al. (2003) Identification of a vitamin $\mathrm{D}$ response element in the human insulin receptor gene promoter. J Steroid Biochem Mol Biol 84, 223-230.

12. Maestro B, Campion J, Davila N et al. (2000) Stimulation by 1,25-dihydroxyvitamin $\mathrm{D}_{3}$ of insulin receptor expression and insulin responsiveness for glucose transport in U-937 human promonocytic cells. Endocr J 47, 383-391.

13. Pittas AG, Lau J, Hu FB et al. (2007) The role of vitamin D and calcium in type 2 diabetes. A systematic review and meta-analysis. J Clin Endocrinol Metab 92, 2017-2029.

14. Johnson JA, Grande JP, Roche PC et al. (1994) Immunohistochemical localization of the $1,25(\mathrm{OH})_{2} \mathrm{D}_{3}$ receptor and calbindin D28k in human and rat pancreas. Am J Physiol 267, E356-E360.

15. Bland R, Markovic D, Hills CE et al. (2004) Expression of 25 -hydroxyvitamin $\mathrm{D}_{3}-1 \alpha$-hydroxylase in pancreatic islets. J Steroid Biochem Mol Biol 89-90, 121-125.

16. Alvarez JA \& Ashraf A (2010) Role of vitamin D in insulin secretion and insulin sensitivity for glucose homeostasis. Int J Endocrinol 2010, 351385.

17. Korea Centers for Disease Control and Prevention, The Korean Pediatric Society, The Committee for the Development of Growth Standard for Korean Children and
Adolescents (2008) 2007 Korean Children and Adolescents Growth Standard. http://www.cdc.go.kr/CDC/cms/cmsFile Download.jsp?fid $=28 \&$ cid $=1235 \&$ fieldName $=$ attach $1 \&$ index $=1$ (accessed November 2012).

18. US Department of Health and Human Services (2011) 2008 Physical activity guidelines for Americans: be active, healthy, and happy! http://www.health.gov/paguidelines (accessed February 2013).

19. Korea Centers for Disease Control and Prevention (2009) The Statistics of 5th Korea Youth Risk Behavior Web-based Survey (KYRBWS) in 2009. http://yhs.cdc.go.kr/ (accessed November 2012).

20. Sempos CT, Vesper HW, Phinney KW et al. (2012) Vitamin D status as an international issue: national surveys and the problem of standardization. Scand J Clin Lab Invest Suppl 243, 32-40.

21. Matthews DR, Hosker JP, Rudenski AS et al. (1985) Homeostasis model assessment: insulin resistance and $\beta$-cell function from fasting plasma glucose and insulin concentrations in man. Diabetologia 28, 412-419.

22. Katz A, Nambi SS, Mather K et al. (2000) Quantitative insulin sensitivity check index: a simple, accurate method for assessing insulin sensitivity in humans. J Clin Endocrinol Metab 85, 2402-2410.

23. Wells JC (2001) A critique of the expression of paediatric body composition data. Arch Dis Child 85, 67-72.

24. Cole TJ \& Green PJ (1992) Smoothing reference centile curves: the LMS method and penalized likelihood. Stat Med 11, 1305-1319.

25. Del Gobbo LC, Song Y, Dannenbaum DA et al. (2011) Serum 25-hydroxyvitamin D is not associated with insulin resistance or $\beta$ cell function in Canadian Cree. J Nutr $\mathbf{1 4 1}$, 290-295.

26. Rajakumar K, de las Heras J, Lee $\mathrm{S}$ et al. (2012) 25 -Hydroxyvitamin $\mathrm{D}$ concentrations and in vivo insulin sensitivity and $\beta$-cell function relative to insulin sensitivity in black and white youth. Diabetes Care 35, 627-633.

27. Pacifico L, Anania C, Osborn JF et al. (2011) Low $25(\mathrm{OH}) \mathrm{D}_{3}$ levels are associated with total adiposity, metabolic syndrome, and hypertension in Caucasian children and adolescents. Eur J Endocrinol 165, 603-611.

28. Rajakumar K, de las Heras J, Chen TC et al. (2011) Vitamin D status, adiposity, and lipids in black American and Caucasian children. J Clin Endocrinol Metab 96, 1560-1567.

29. Golden SH, Brown A, Cauley JA et al. (2012) Health disparities in endocrine disorders: biological, clinical, and nonclinical factors - an Endocrine Society scientific statement. J Clin Endocrinol Metab 97, e1579-e1639.

30. Chan JC, Malik V, Jia W et al. (2009) Diabetes in Asia: epidemiology, risk factors, and pathophysiology. JAMA 301, 2129-2140.

31. Holick MF, Binkley NC, Bischoff-Ferrari HA et al. (2011) Evaluation, treatment, and prevention of vitamin D deficiency: an Endocrine Society clinical practice guideline. J Clin Endocrinol Metab 96, 1911-1930.

32. Kremer R, Campbell PP, Reinhardt T et al. (2009) Vitamin D status and its relationship to body fat, final height, and peak bone mass in young women. J Clin Endocrinol Metab 94, 67-73.

33. Hatun S, Islam O, Cizmecioglu F et al. (2005) Subclinical vitamin D deficiency is increased in adolescent girls who wear concealing clothing. J Nutr 135, 218-222. 